\title{
Age-related reduction of dermal fibroblast size upregulates multiple matrix metalloproteinases as observed in aged human skin in vivo
}

\author{
Z. Qin, R.M. Balimunkwe and T. Quan \\ Department of Dermatology, University of Michigan Medical School, Ann Arbor, MI, U.S.A.
}

Linked Comment: Hibbert. Br J Dermatol 2017; 177:1160-1161.

\section{Summary}

\section{Correspondence \\ T. Quan. \\ E-mail: thquan@umich.edu}

Accepted for publication

7 February 2017

Funding sources:

National Institute of Health (AG19364).

Conflicts of interest:

None declared.

Z.Q. and R.M.B. contributed equally to this work.

DOI $10.1111 /$ bjd.15379
Background Fragmentation of collagen fibrils, the major structure protein in skin, is a hallmark of dermal ageing. Matrix metalloproteinases (MMPs) are largely responsible for the fragmentation of collagen fibrils.

Objectives To quantify gene expression of all 23 known mammalian MMPs in sunprotected young and aged human skin in vivo and to investigate the potential mechanism underlying age-related alteration of multiple MMPs.

Methods MMP mRNA expression levels and MMP activity in sun-protected young and aged human skin in vivo were determined by real-time reverse transcription polymerase chain reaction (RT-PCR) and in situ zymography, respectively. The relative contributions to elevated MMPs in epidermis and dermis were quantified by laser capture microdissection coupled real-time RT-PCR. Dermal fibroblast morphology and collagen fibril fragmentation in human skin in vivo were assessed by second-harmonic generation microscopy and atomic force microscopy, respectively. In vitro cell morphology was assessed by CellTracker ${ }^{\circledR}$ fluorescent dye (Molecular Probes, Eugene, OR, U.S.A.) and phalloidin staining. Protein levels were determined by ProteinSimple capillary electrophoresis immunoassay (ProteinSimple, Santa Clare, CA, U.S.A.).

Results Multiple MMPs are elevated in aged human skin dermis. Increased MMP activity and collagen fibril fragmentation were observed in aged skin dermis. As dermal fibroblasts are the major MMP-producing cells in the dermis, reduction of dermal fibroblast size, which is observed in aged human skin, contributes to the elevation of age-related multiple MMPs. Reduction of fibroblast size upregulates C-Jun/C-Fos and activates AP-1.

Conclusions Combined actions of the wide variety of MMPs that are constitutively elevated in aged dermis may be involved in the progressive degradation of dermal collagen fibrils. Age-related elevations of multiple MMPs are likely to be a result of the reduction of fibroblast size via activation of AP-1.

\section{What's already known about this topic?}

- Collagen is the major structural protein in skin, and its fragmentation is a hallmark of dermal ageing.

- Matrix metalloproteinases (MMPs) are largely responsible for the fragmentation of collagen fibrils.

- However, the expression of all known mammalian MMPs and the mechanism underlying the altered expression of MMPs in chronologically aged human skin are not well understood.

\section{What does this study add?}

- Multiple MMPs are elevated in aged human skin dermis. 
- As dermal fibroblasts are the major MMP-producing cells in the dermis, the reduction of dermal fibroblast size contributes to the elevation of age-related multiple MMPs.

- Reduction of fibroblast size upregulates c-Jun/c-Fos and activates AP-1.

- These findings provide a foundation for understanding the cellular and molecular basis of age-related collagen fragmentation, the characteristic feature of aged human skin.

\section{What is the translational message?}

- Enhancing the structural integrity of dermal collagen and fibroblast size prevents age-related elevations of multiple MMPs, which may improve health, function and wound healing in the elderly.

Collagen is the most abundant protein in mammals, making up about one-third of the whole-body protein content. ${ }^{1}$ In human skin, collagen is the main component of the dermis that provides structural and functional support of the skin. ${ }^{2}$ During ageing, dermal collagen fibrils undergo progressive alterations owing to age-related fragmentation and disorganization of collagen fibrils. ${ }^{3,4}$ Fragmentation of collagen fibrils is essentially responsible for aged-appearing skin and the most visible signs of ageing such as wrinkles and fragile atrophic skin. Alterations of dermal collagen impair skin structural integrity and are associated with age-related disorders, such as increased fragility, ${ }^{5}$ impaired vasculature support, ${ }^{6,7}$ poor wound healing ${ }^{8,9}$ and the promotion of skin cancer. ${ }^{10,11}$

In human skin, dermal fibroblasts are responsible for collagen homeostasis. ${ }^{12}$ Dermal fibroblasts are embedded in a collagen-rich microenvironment and physically interact with collagen fibrils to maintain normal cell shape and size. The accumulating evidence demonstrates that cell shape and size regulate essential cell functions. ${ }^{13,14}$ Cell shape and size are largely regulated by interactions of cells with the surrounding extracellular matrix (ECM). The ECM provides both binding sites for cells and mechanical resistance to cellular traction forces. ${ }^{14,15}$ In young healthy skin, dermal fibroblasts interact with intact collagen fibrils and exert traction forces to achieve normal cell shape and size. However, in aged dermis, collagen fibrils are fragmented, which impairs fibroblast-collagen interaction and results in reduced fibroblast spreading, shape and size. ${ }^{3,16,17}$ While cell shape and size are known to regulate many cellular functions, the molecular basis of their impact on dermal fibroblast function and dermal ageing are not well understood.

Matrix metalloproteinases (MMPs) are a large family of proteinases that are essentially responsible for the fragmentation of collagen fibrils. ${ }^{18,19}$ We previously reported that dermal fibroblasts in aged human skin express elevated MMP-1, which leads to the initiation of collagen fibril fragmentation. ${ }^{3,20}$ However, the complete profile of all 23 known mammalian MMPs in aged human skin is unknown. Here, we investigate the expression of all 23 known mammalian MMPs in sun-protected young and aged human skin in vivo. We found that multiple MMPs are constitutively elevated in aged human skin dermis. The reduction of fibroblast size is a prominent feature of aged human skin. ${ }^{3,16,17}$ We found that the reduction of fibroblast size activates transcription factor AP-1, the major regulator of multiple MMPs, ${ }^{21-23}$ leading to the upregulation of multiple age-related MMPs observed in aged human skin. Our data suggest insights into the mechanisms of collagen fibril fragmentation, which is a defining feature of the pathophysiology of human dermal ageing.

\section{Materials and methods}

\section{Procurement of human skin samples and laser capture microdissection}

All procedures involving human subjects were approved by the University of Michigan Institutional Review Board and all subjects provided written informed consent. Human skin punch biopsies from sun-protected buttock skin were obtained from clinically normal adult volunteers; 25-30 years of age for young group ( $n=12$, mean age $26 \pm 3$ years; men) and $\geq 80$ years of age for the aged-skin group ( $n=12$, mean age $83 \pm 4$ years). Skin samples were $4 \mathrm{~mm}$ in diameter (full thickness skin). To quantify the relative levels of epidermal and dermal MMPs, epidermis and dermis were captured by laser capture microdissection (LCM) $(15 \mu \mathrm{m})$, and were collected in lysis buffer (RNeasy Micro kit, Qiagen, Chatsworth, CA, U.S.A.), followed by RNA extraction and real-time reverse transcription polymerase chain reaction (RT-PCR), as described below.

\section{Cell culture and three-dimensional collagen lattice cell cultures}

Young (21-30 years) and aged ( $>80$ years) human skin primary dermal fibroblasts were isolated from punch biopsies of sun-protected buttock skin by digestion skin with bacterial collagenase (Worthington Biochemical Corporation, 
Lakewood, NJ, U.S.A.). Cells were maintained in Dulbecco's modified Eagle's medium supplemented with 10\% fetal bovine serum (Invitrogen, Carlsbad, CA, U.S.A.), penicillin (100 $\left.\mathrm{U} \mathrm{mL}^{-1}\right)$ and streptomycin $\left(100 \mu \mathrm{gL}^{-1}\right)$ in a humidified incubator with $5 \% \mathrm{CO}_{2}$ at $37{ }^{\circ} \mathrm{C}$. Cells were utilized between passages 4 and 9 , and replication experiments used fibroblasts from different donors. In some cases, dermal fibroblasts were cultured in three-dimensional (3D) collagen lattices, based on a previous publication with minor modification. ${ }^{17}$ Briefly, neutralized collagen lattices were prepared by mixing the appropriate volume of rat tail type I collagen (BD Biosciences San Jose, CA, U.S.A.) with medium cocktail [Dulbecco's Modified Eagle Medium (DMEM), NaHCO3 (44 mmol L ${ }^{-1}$ ), L-glu-

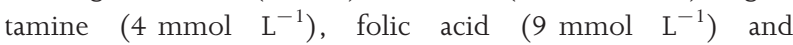
neutralized with $1 \mathrm{~N} \mathrm{NaOH}$ to $\mathrm{pH}$ 7.2] to yield a final concentration of $2 \mathrm{mg} \mathrm{mL}^{-1}$. Next, $0.5 \times 10^{6}$ cells were suspended in $2 \mathrm{~mL}$ collagen solution in a $35-\mathrm{mm}$ culture dish, and placed in an incubator at $37{ }^{\circ} \mathrm{C}$ for $30 \mathrm{~min}$ to allow polymerization of the collagen. For the constrained culture system, cells were cultured in collagen gel in which a nylon mesh $(0.5 \mathrm{~mm}$ pore size) was placed on the bottom of the culture dish to provide cytoskeletal stability and tension. For the unconstrained culture system, cells were cultured in collagen lattices without nylon mesh. The collagen gels were then incubated with $2 \mathrm{~mL}$ media (DMEM, 10\% fetal bovine serum) at $37{ }^{\circ} \mathrm{C}, 5 \% \mathrm{CO}_{2}$. For analyses, cells were harvested by digesting collagen gel with bacterial collagenase ( $1 \mathrm{mg} \mathrm{mL}^{-1}$, Sigma, St. Louis, MO, U.S.A.) for $30 \mathrm{~min}$ at $37^{\circ} \mathrm{C}$. Fibroblasts were collected by centrifugation and recovery of viable fibroblasts $(>80 \%)$ was confirmed by trypan blue staining. For latrunculin (Lat)-A treatment, cells were treated with Lat-A at a concentration of $30 \mathrm{nmol} \mathrm{L}^{-1}$ for $24 \mathrm{~h}$ or the indicated times.

\section{RNA isolation, laser capture microdissection and quantitative reverse transcription polymerase chain reaction}

Total RNA was extracted, using a commercial kit (RNeasy midikit; Qiagen), and reverse transcription was performed using a Taqman Reverse Transcription kit (Applied Biosystems, Foster City, CA, U.S.A.). Real-time RT-PCR was performed using a Taqman Universal PCR Master Mix kit (Applied Biosystems) and 7300 Real-Time PCR System (Applied Biosystems). PCR procedures were performed using a robotic workstation (Biomek 2000; Beckman Coulter Inc., Hialeah, FL, U.S.A.) to ensure accuracy and reproducibility. All primers and probes were purchased from Applied Biosystems (Assayson-Demand $^{\mathrm{TM}}$ Gene Expression Products). Multiplex PCR reactions contained primers and probes for the target gene and 36B4, a ribosomal protein used as an internal normalization control for quantitation.

\section{ProteinSimple capillary electrophoresis immunoassay}

Dermal fibroblasts were lysed in whole-cell extraction buffer [25 mmol L ${ }^{-1}$ HEPES (pH 7.7); $0.3 \mathrm{~mol} \mathrm{~L}^{-1} \mathrm{NaCl} ; 1.5 \mathrm{mmol}$
$\mathrm{L}^{-1} \mathrm{MgCl}_{2} ; 0.2 \mathrm{mmol} \mathrm{L} \mathrm{L}^{-1}$ ethylenediaminetetraacetic acid; $0 \cdot 1 \%$ Triton X-100; 0.5 mmol L ${ }^{-1}$ dithiothreitol (DTT); $20 \mathrm{mmol} \mathrm{L}{ }^{-1} \beta$-glycerolphosphate; $0 \cdot 1 \mathrm{mmol} \mathrm{L} \mathrm{L}^{-1} \quad \mathrm{Na}_{3} \mathrm{VO}_{4}$; $2 \mu \mathrm{g} \mathrm{mL}^{-1}$ leupeptin; and $100 \mu \mathrm{g} \mathrm{mL}^{-1}$ phenylmethylsulfonyl fluoride]. Whole-cell extracts were prepared by centrifugation and protein concentration was determined by the BioRad protein assay (BioRad Laboratories, Hercules, CA, U.S.A.). ProteinSimple capillary electrophoresis immunoassay (ProteinSimple, Santa Clare, CA, U.S.A.) was performed according to the ProteinSimple user manual. In brief, whole-cell extract samples (800 ng per lane) were mixed with a master mix (ProteinSimple) to a final concentration of $1 \times$ sample buffer, $1 \times$ fluorescent molecular weight markers, and $40 \mathrm{mmol} \mathrm{L}^{-1}$ DTT and then heated at $95{ }^{\circ} \mathrm{C}$ for 5 min. The samples, blocking reagent, primary antibodies, horseradish peroxidase-conjugated secondary antibodies, chemiluminescent substrate and separation and stacking matrices were also dispensed to a designated wells plate. The electrophoresis and immunodetection steps took place in the capillary system and were fully automated using instrument default settings. The digital image was analysed and quantified with Compass software (ProteinSimple) after normalization using $\beta$-actin (loading control).

\section{CellTracker, phalloidin staining, immunohistology and second-harmonic generation microscopy}

Cell morphology in 3D collagen lattices was assessed by incubation of cultures with CellTracker ${ }^{\circledR}$ fluorescent dye (Molecular Probes, Eugene, OR, U.S.A.) for $1 \mathrm{~h}$. The cells were washed with phosphate-buffered saline (PBS) and were fixed in $2 \%$ paraformaldehyde for $30 \mathrm{~min}$. Cell morphology in monolayer culture was assessed by phalloidin staining. Cells were washed with PBS and were fixed in 2\% paraformaldehyde for $30 \mathrm{~min}$ followed by phalloidin staining (Sigma, St. Louis, MO, U.S.A.) for $1 \mathrm{~h}$. Images were obtained using Zeiss fluorescence microscopy. Second-harmonic generation microscopy was performed using a Leica SP8 Confocal Microscope with 2-Photon at University of Michigan Microscopy and Image Analysis Laboratory.

\section{Transfection AP-1 reporter activity assay}

Primary adult human dermal fibroblasts were transiently transfected with AP-1 reporter construct (pAP1-TA-Luc) purchased from BD Biosciences Clontech (Palo Alto, CA, U.S.A.). Cells were transfected by electroporation using human dermal fibroblasts nucleofector kit (Amaxa Biosystems, Gaithersburg, $\mathrm{MD}$, U.S.A.) according to the manufacturer's protocol. After $48 \mathrm{~h}$ of transfection, AP-1 luciferase activity was measured by luciferase assay using an enhanced luciferase assay kit (PharMingen International, San Diego, CA, U.S.A.) according to the manufacturer's protocol. The $\beta$-galactosidase expression vector was used as an internal control for transfection efficiency, and aliquots containing identical $\beta$-galactosidase activity were used for each luciferase assay. 


\section{Zymography}

In situ zymography was performed as described previously. ${ }^{24}$ Briefly, frozen skin sections were placed on glass slides coated with fluorescently labelled collagen (Elastin products, Owensville, MO, U.S.A.) as substrate. Skin sections were incubated for $24 \mathrm{~h}$ to allow MMPs in the tissue to degrade the fluorescein-labelled collagen in the slide.

\section{Atomic force microscopy imaging}

Optimal cutting temperature embedded human skin samples were sectioned $(50 \mu \mathrm{m})$ and mounted on glass coverslips (1.2 mm diameter; Fisher Scientific Co., Pittsburgh, PA, U.S.A.). These atomic force microscopy (AFM) samples were allowed to air-dry for at least $24 \mathrm{~h}$ before AFM analysis. ${ }^{25}$ The scan positions of the collagen lattices were determined by light optical image. Images were obtained by AFM with ScanAsyst mode (Dimension Icon, Bruker-AXS, Santa Barbara, CA, U.S.A.) in air using a silicon-etched cantilever (NSC15/AIBS, MikroMasch, San Jose, CA, U.S.A.) with a full tip cone angle $\sim 40^{\circ}$ and the tip radius of curvature $\sim 10 \mathrm{~nm}$. AFM images were acquired at a scan rate of $0.977 \mathrm{~Hz}(512 \times 512$ pixel resolutions). AFM imaging was conducted at the Electron Microbeam Analysis Laboratory, University of Michigan College of Engineering, and analysed using Nanoscope Analysis software (Nanoscope Analysis v120R1sr3, Bruker-AXS, Santa Barbara, CA, U.S.A.).

\section{Statistical analysis}

Statistical significance between groups was determined with the Student's t-test. All P-values are two-tailed and considered significant when $\mathrm{P}<0.05$.

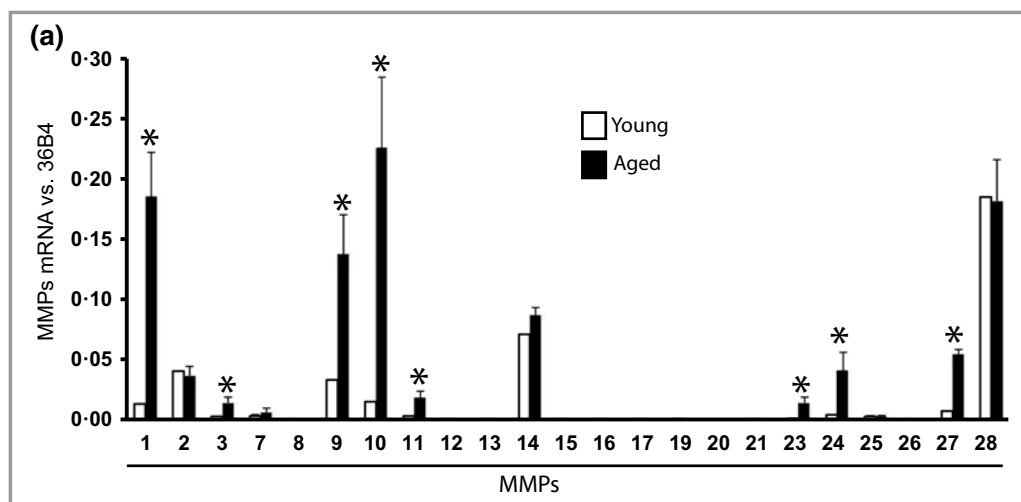

(b)

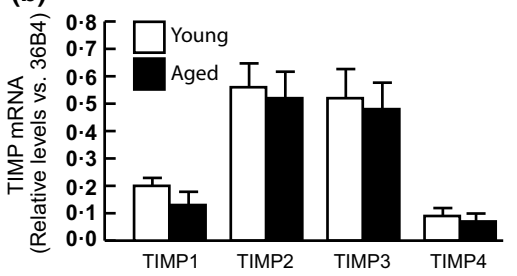

(c)

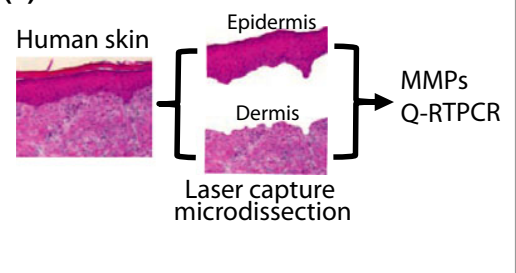

(d)

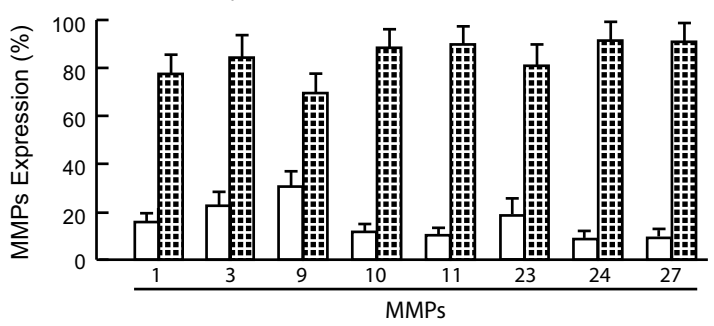

(e)
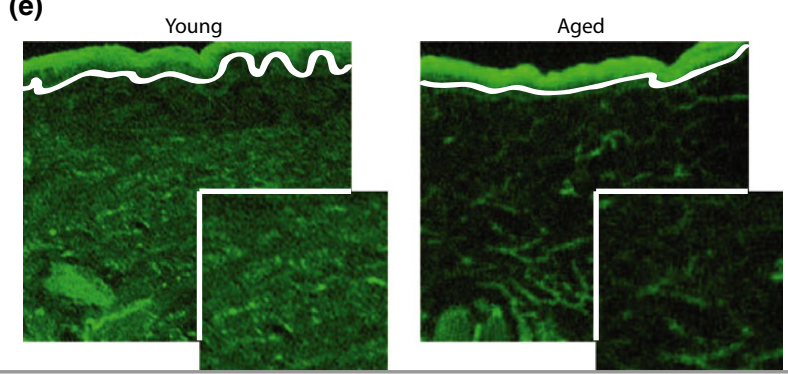

Fig 1. Elevated expression of multiple matrix metalloproteinases (MMPs) in aged human dermis. Adult human skin punch biopsies were obtained from sun-protected buttock skin. (a) Elevated multiple MMPs in aged (83 \pm 4 years) human skin compared with young ( $26 \pm 3$ years) human skin ( $n=12$ in each group) (mean \pm SEM). $* \mathrm{P}<0.05$. (b) No change in tissue inhibitors of metalloproteinase mRNA expression in young and aged human skin ( $\mathrm{n}=12$ in each group) (mean \pm SEM). (c) Schematic representation of the dissection of human skin epidermis and dermis by laser capture microdissection (LCM) (see Materials and methods section for details). (d) Elevated MMPs in the dermis of aged human skin. Epidermis and dermis were captured by LCM and total RNA was prepared from epidermis and dermis $(\mathrm{n}=8)$ (mean \pm SEM). All MMP mRNA levels were quantified by real-time reverse transcription polymerase chain reaction (Q-RTPCR) and were normalized by the housekeeping gene (36B4, internal control). (e) Elevated collagenase activity in the dermis of aged human skin determined by in situ zymography (see Materials and methods section for details). Loss of green fluorescence in aged dermis indicates degradation of fluorescein collagen substrate. White lines indicate the boundary between the epidermis (top) and dermis (bottom) $(n=6)$. TIMP, tissue inhibitor of metalloproteinase. 


\section{Results}

\section{Elevation of multiple matrix metalloproteinases in aged human skin dermis in vivo}

Measurement of all 23 known mammalian MMPs indicated that eight MMPs were significantly elevated in sun-protected (buttock) aged ( $83 \pm 4$ years) human skin, compared with sun-protected young (26 \pm 3 years) skin (relative fold increase from high to low: MMP-10, MMP-1, MMP-9, MMP27, MMP-24, MMP-11, MMP-3, MMP-23) (Fig. 1a). MMPs and tissue inhibitors of metalloproteinases (TIMPs) are often coordinately regulated as a means to control excess MMP activity; we also investigated whether TIMPs are elevated in aged skin. However, no differences in mRNA levels of all four known TIMPs were found between young and aged human skin (Fig. 1b). To quantify the relative contributions to elevated MMPs, epidermis and dermis were separated by LCM
Fig 2. (a) Multiple matrix metalloproteinases (MMPs) are similarly expressed in the fibroblasts (FBs) isolated from aged (83 \pm 4 years) and young (26 \pm 3 years) skin. Dermal fibroblasts were isolated from young and aged sun-protected buttock skin. MMP mRNA expression levels were quantified by real-time reverse transcription polymerase chain reaction and were normalized by the housekeeping gene (36B4, internal control) ( $\mathrm{n}=6$, mean \pm SEM). (b) Reduced dermal fibroblast size is a prominent feature of aged dermal fibroblasts in human skin in vivo. Representative images of dermal fibroblasts in aged (78 years, right panel) and young (26 years, left panel) human skin. Skin was sectioned and dermal fibroblasts were identified by immunostaining with collagen chaperone heat shock protein 47 (blue). Grey/white colour indicates collagen fibrils and green colour indicates elastin. Note the spread fibroblasts in young skin vs. contracted fibroblasts in aged human skin. Images were obtained by multiphoton laser scanning fluorescence microscopy ( $\mathrm{n}=6$ for each group). The black and white arrowheads indicate dermal fibroblasts in young and aged human skin, respectively. Scale bar $=25 \mu \mathrm{m}$. (c) Collagen fibrils in aged skin dermis were fragmented and disorganized. Nanoscale collagen fibrils were imaged by atomic force microscopy. The black and white arrowheads indicate intact and fragmented/disorganized collagen fibrils, respectively. Images are representative of six independent experiments. Scale bar $=100 \mathrm{~nm}$. (d) The morphology of the dermal fibroblasts from young (left panel) and aged (right panel) skin were similar in standard monolayer culture in vitro. Cells were stained with CellTracker ${ }^{\circledR}$ fluorescent dye (Molecular Probes, Eugene, OR, U.S.A.) and were imaged by fluorescence microscopy. Red fluorescence delineates cell cytoplasm; blue fluorescence delineates nuclei. Scale bar $=$ $100 \mu \mathrm{m}$. Images are representative of the dermal fibroblasts from five young and aged individuals.

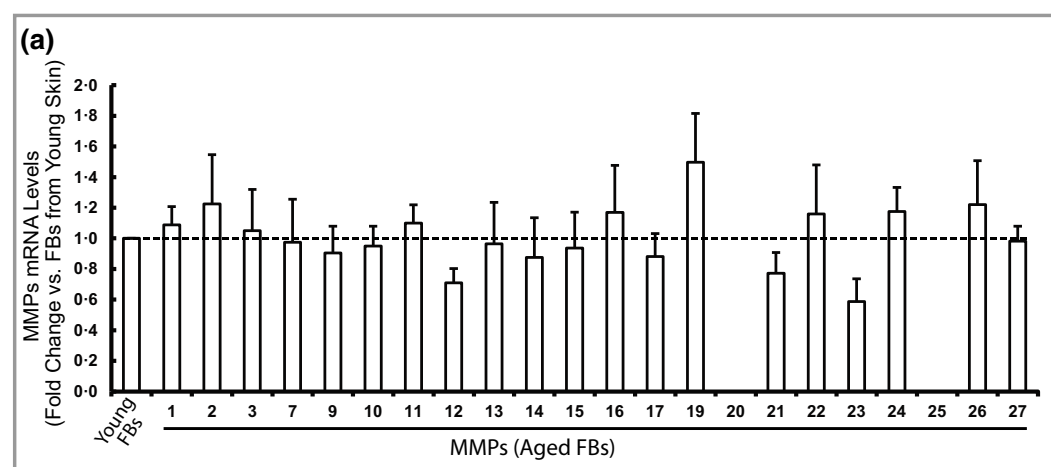

(b)
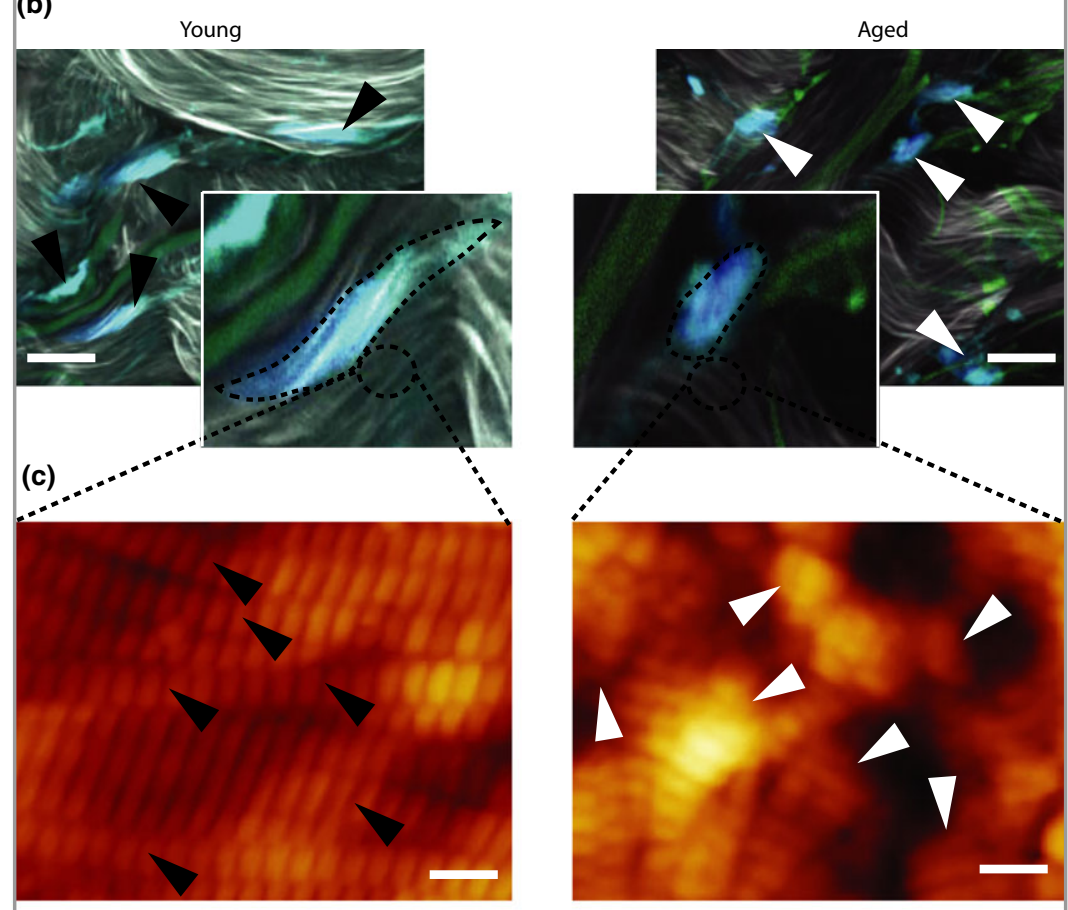

(d)

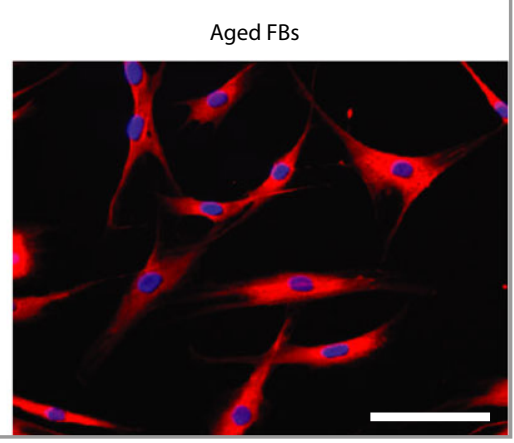



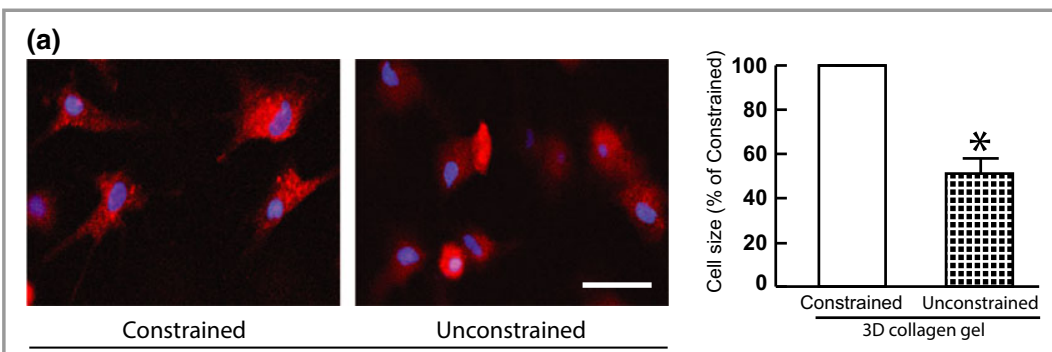

(b)

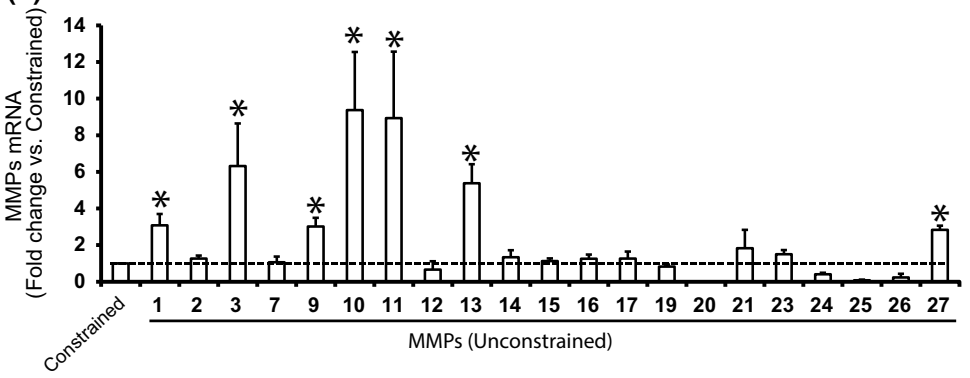

(c)
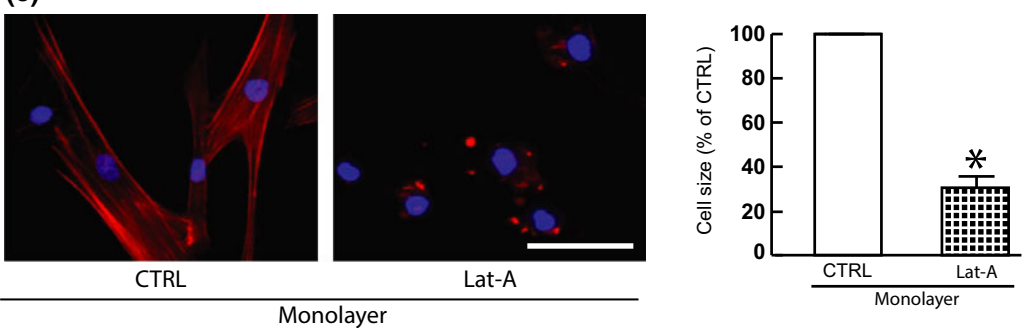

(d)

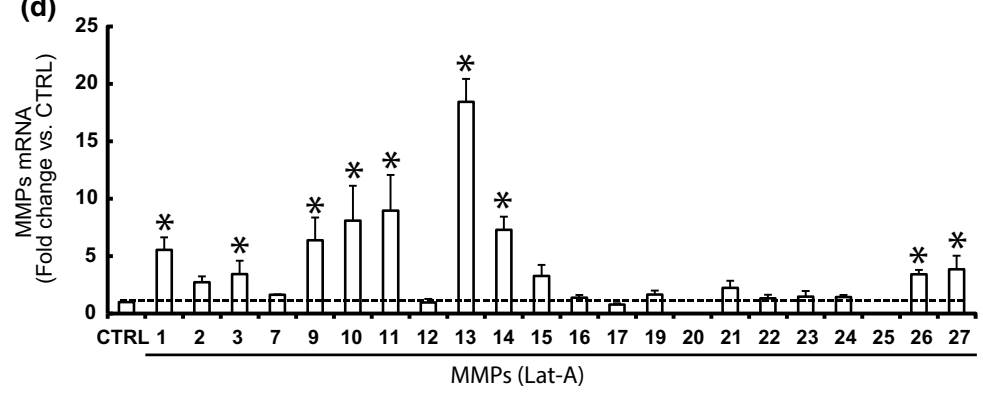

(e)

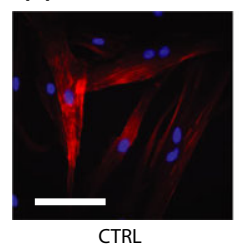

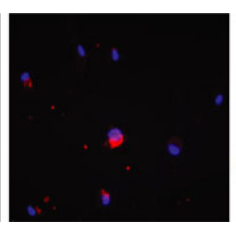

Lat-A

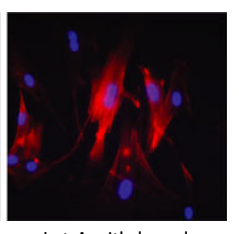

Lat-A withdrawal

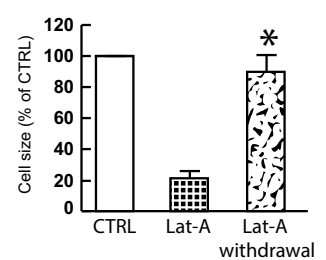

(f)

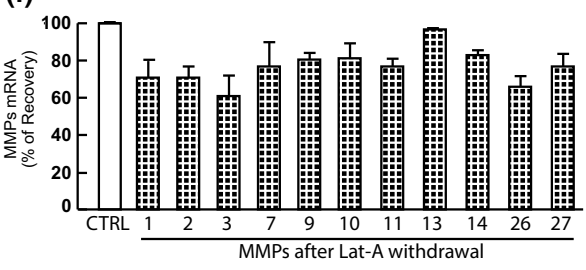

(Fig. 1c). Figure 1(d) shows that all MMPs that are elevated in aged skin were primarily expressed in the dermis [MMP-10 (88\%), MMP-1 (78\%), MMP-9 (70\%), MMP-27 (91\%),
MMP-24 (91\%), MMP-11 (92\%), MMP-3 (84\%) and MMP$23(81 \%)]$. To access MMP activity, we performed in situ zymography, in which unfixed skin sections are placed over a 
Fig 3. Age-related reduction of dermal fibroblast size upregulates multiple matrix metalloproteinases (MMPs) observed in aged human skin. (a) To model young and aged dermal fibroblasts, dermal fibroblasts from young skin ( $26 \pm 3$ years) were cultured under conditions of constrained (left panel) and unconstrained (right panel) 3D collagen lattices (see Materials and methods section for details). Cells were stained with CellTracker® fluorescent dye (Molecular Probes, Eugene, OR, U.S.A.) and were imaged by fluorescence microscopy. Red fluorescence delineates cell cytoplasm; blue fluorescence delineates nuclei. Relative cell surface areas were quantified using ImageJ software and data were expressed as a percentage of the control (noncontracted gel) $(n=3$, mean \pm SEM). $* \mathrm{p}<0 \cdot 05$. Scale bars $=100 \mu \mathrm{m}$. (b) The majority of age-related MMPs were elevated by the reduction of fibroblast size. Total RNA was extracted from dermal fibroblasts cultured from 3D collagen lattices. MMP mRNA expression levels were quantified by real-time reverse transcription polymerase chain reaction (RT-PCR) and were normalized by the housekeeping gene (36B4, internal control) $(\mathrm{n}=4$, mean $\pm \mathrm{SEM}) .{ }^{*} \mathrm{P}<0 \cdot 05$. (c) Dermal fibroblasts from young skin $(26 \pm 3$ years) were treated with latrunculin (Lat)-A $\left(30 \mathrm{nmol} \mathrm{L}^{-1}\right)$ for $24 \mathrm{~h}$. Dermal fibroblasts were stained with phalloidin and were imaged by fluorescence microscopy. Red fluorescence delineates cell cytoplasm; blue fluorescence delineates nuclei. Relative cell surface areas were quantified using ImageJ software and data were expressed as a percentage of the control [dimethylsulfoxide (DMSO)] $(\mathrm{n}=6$, mean \pm SEM). $* \mathrm{P}<0 \cdot 05$. Scale bar $=100 \mu$ m. $(\mathrm{d})$ The majority of age-related MMPs were elevated by the reduction of fibroblast size via disruption of the actin cytoskeleton. Total RNA was extracted from dermal fibroblasts. MMP mRNA expression levels were quantified by real-time RT-PCR and were normalized by the housekeeping gene (36B4, internal control). Data are mean $\pm \operatorname{SEM}(n=5) . * P<0 \cdot 05$. (e) Restoration of dermal fibroblast size after withdrawal of Lat-A. Lat-A was withdrawn (right panel) $24 \mathrm{~h}$ after Lat-A $\left(30 \mathrm{nmol} \mathrm{L}^{-1}\right.$ ) treatment (middle panel) by replacing with fresh culture medium, and the cells were then further incubated for $48 \mathrm{~h}$. Dermal fibroblasts were stained with phalloidin and relative cell surface areas were quantified using ImageJ software. Data were expressed as percentage of the control (DMSO, left panel). Data are mean \pm SEM $(n=6)$. $* P<0 \cdot 05$. Scale bars $=100 \mu$ m. (f) Restoration of dermal fibroblast size reverses elevated MMPs. MMP mRNA expression levels were quantified by real-time RT-PCR and were normalized by the housekeeping gene (36B4, internal control). MMPs mRNA expression levels after Lat-A was withdrawn were expressed as a percentage of the control cells (DMSO). Data are mean \pm SEM $(n=5)$.

layer of fluorescently labelled collagen. As shown in Figure 1(e), elevated MMP activity in aged skin resulted in the breakdown of the collagen, resulting in loss of fluorescence. These data demonstrate that multiple MMPs are elevated in sun-protected aged human skin dermis, suggesting that the combined actions of the wide variety of MMPs may contribute to the degradation of dermal collagen fibrils.

\section{Age-related reduction of dermal fibroblast size upregulates multiple matrix metalloproteinases observed in aged human skin}

Next, we investigated the potential mechanisms of age-related elevation of multiple MMPs using primary dermal fibroblasts, as dermal fibroblasts are the major MMP-producing cells in the dermis and are primarily responsible for collagen turnover. To this end, we first isolated the dermal fibroblasts from sunprotected young human skin (mean age $26 \pm 3$ years) and aged human skin (mean age $83 \pm 4$ years) and examined the mRNA expression of all known mammalian MMPs in standard monolayer culture. Interestingly, we found that all 23 MMPs are similarly expressed in the fibroblasts isolated from young and aged skin (Fig. 2a). These data suggest that the agerelated elevation of multiple MMPs might be induced by epigenetics rather than intrinsic genetic alteration.

We previously reported that reduced size is a prominent characteristic of aged dermal fibroblasts in human skin in vivo, with decreased elongation and a more rounded, collapsed morphology owing to collagen fragmentation. ${ }^{3,16,17}$ These data prompted us to explore the connection between reduced fibroblast size and age-related elevation of multiple MMPs. Using second-harmonic generation microscopy, we confirmed that the fibroblasts in aged dermis revealed reduced size and collapsed morphology (Fig. 2b, upper right panel), compared with elongated and stretched fibroblasts from young skin dermis (Fig. 2b, upper left panel). AFM indicated that collagen fibrils in young skin dermis were intact, tightly packed and well organized (Fig. 2c, lower left panel). In contrast, collagen fibrils in aged skin dermis were fragmented, sparse and disorganized (Fig. 2c, lower right panel), suggesting that fragmented and disorganized collagen fibrils appear to be unable to support normal fibroblast morphology. Consequently, we confirmed that the morphology of the isolated dermal fibroblasts from young (Fig. 2d, left panel) and aged skin (Fig. 2d, right panel) were similar in standard monolayer culture in vitro, suggesting that the dermal ECM microenvironment is largely responsible for fibroblast shape and size.

Based on the above observations, we investigated the relationship between reduced fibroblast size and age-related elevation of multiple MMPs using young dermal fibroblasts (mean age $26 \pm 3$ years). Firstly, we modulated dermal fibroblast size by culturing young dermal fibroblasts in 3D collagen lattices, using constrained and unconstrained 3D collagen lattices to model young and aged dermal fibroblasts, respectively. A red fluorescent dye (CellTracker), which is taken up into the cell cytoplasm, was used to assess the morphology of fibroblasts in 3D collagen lattices. Dermal fibroblasts cultured in constrained collagen lattices displayed a spread flattened appearance (Fig. 3a, left). In contrast, fibroblasts in unconstrained collagen gels had reduced cytoplasmic area and a contracted appearance (Fig. 3b, right), similar to fibroblasts in aged human skin in vivo (Fig. 2b, upper right panel). Quantification indicated that fibroblast size was reduced by approximately $50 \%$ in unconstrained collagen gel (Fig. 3b, right panel). Interestingly, the measurement of all 23 mammalian MMPs indicated that the majority of MMPs that were found to be elevated in aged dermis in vivo (except for MMP-23 and MMP-24) were elevated by the reduction of 
fibroblast size (Fig. 3b). To obtain further confirmation of this fibroblast-size-dependent elevation of MMPs, we modulated young dermal fibroblast size by disrupting the actin cytoskeleton with Lat-A, which rapidly blocks actin polymerization. ${ }^{26}$ As expected, the disruption of the actin cytoskeleton resulted in loss of actin cytoskeletal fibres and reduced fibroblast size by approximately 70\% (Fig. 3c, right panel). Importantly, the reduction of fibroblast size resulted in the elevation of multiple MMPs that were found to be elevated in aged dermis in vivo (Fig. 2e). As shown in Table 1, the reduction of fibroblast size, in either $3 \mathrm{D}$ collagen lattices or in monolayer culture, resulted in the elevation of the majority of MMPs that were elevated in aged dermis in vivo, except MMP-23 and MMP-24.

Next, we assessed whether elevated MMPs are reversible by restoration of fibroblast size. For these studies, media containing Lat-A was withdrawn $24 \mathrm{~h}$ after its addition, and the cells were extensively washed. Fresh culture media was then added. Following the removal of Lat-A, fibroblasts converted from a small rounded morphology (Fig. 3e, middle panel) to a typical elongated morphology (Fig. 3e, right panel). Consistent with the recovery of cellular size, the elevated MMPs were normalized to basal level (Fig. 3f). These data suggest that reduced fibroblast size as observed in aged human skin likely contributes to the age-related elevation of multiple MMPs.

Table 1 Age-related reduction of dermal fibroblast size upregulates multiple matrix metalloproteinases (MMPs) as observed in aged human skin

\begin{tabular}{llll}
\hline & \multicolumn{3}{c}{ Reduced cell size } \\
\cline { 2 - 4 } & Aged skin & 3D collagen gel & Monolayer \\
\hline MMP-1 & + & + & + \\
MMP-2 & & & + \\
MMP-3 & + & + & \\
MMP-7 & & & + \\
MMP-8 & & & + \\
MMP-9 & + & + & + \\
MMP-10 & + & + & + \\
MMP-11 & + & + & + \\
MMP-12 & & & \\
MMP-13 & & + & + \\
MMP-14 & & & + \\
MMP-15 & & & \\
MMP-16 & & & + \\
MMP-17 & & & \\
MMP-18 & & & \\
MMP-19 & & & \\
MMP-20 & & & \\
MMP-21 & & & \\
MMP-22 & & & \\
MMP-23 & + & & \\
MMP-24 & + & & \\
MMP-25 & & & \\
MMP-26 & & & \\
MMP-27 & + & & \\
MMP-28 & & & \\
\hline
\end{tabular}

British Journal of Dermatology (2017) 177, pp1337-1348

\section{Reduced fibroblast size upregulates c-Jun/c-Fos and activates AP-1, the major regulator of multiple matrix metalloproteinases}

Next, we investigated the mechanism by which reduced fibroblast size upregulates multiple MMP expression. We focused on transcription factors c-Jun and c-Fos, which typically comprise the AP-1 transcription factor complex. AP-1 transcription factor has been shown to be the principal regulator of multiple MMP transcription under a variety of conditions. ${ }^{21,22,27}$ As shown in Figure 4, the reduction of fibroblast size in either 3D collagen lattices (noncontraction vs. contraction) or monolayer culture [dimethylsulfoxide (DMSO) vs. Lat-A] resulted in significant increases of c-Jun mRNA (Fig. 4a) and protein (Fig. 4b), and c-Fos mRNA (Fig. 4c) and protein (Fig. 4d). Furthermore, the reduction of fibroblast size markedly increased AP-1 reporter activities (Fig. 4e). Finally, we asked whether c-Jun and c-Fos expression are elevated in aged human skin dermis in vivo, which is the major source of elevated multiple MMPs. To address this question, the dermis was prepared by cutting off epidermis at a depth of $1 \mathrm{~mm}$ by cryostat. Indeed, both c-Jun and c-Fos mRNA (Fig. 5a) and protein levels (Fig. 5b) were elevated in aged dermis, compared with young dermis. These data suggest that the reduction of fibroblast size may elevate multiple MMPs via upregulation of c-Jun/c-Fos and activation of AP-1, which is the major regulator of multiple MMPs.

\section{Discussion}

MMPs are primarily responsible for the fragmentation of dermal collagen fibrils, the hallmark of dermal ageing. ${ }^{3,4,12}$ MMPs are a large family of proteinases that are capable of degrading every type of ECM protein. ${ }^{18}$ We demonstrate here that multiple MMPs become elevated during the ageing process in human skin dermis. Elevated MMPs in aged dermis can be divided into the following groups: collagenase (MMP-1), gelatinase-B (MMP-9), stromelysins (MMP-3), MMP-10, MMP-11 and membrane-associated MMPs (MMP-23, MMP-24 and the recently identified MMP-27). It is well-known that ultraviolet (UV) radiation from the sun causes premature skin ageing (photoageing) by transiently inducing only three MMPs in human skin in vivo (MMP-1, MMP-3 and MMP-9). ${ }^{28-30} \mathrm{We}$ demonstrate here that compared with acute UV irradiation, a larger variety of MMPs, including UV-inducible MMPs, are constitutively elevated in aged skin. Interestingly, compared with acute UV irradiation, in which the epidermis is the primary source of transiently induced MMPs, ${ }^{30}$ the dermis is the major source of elevated MMPs in chronologically aged sunprotected skin. These observations suggest that although chronologically aged and photoaged skin share many common molecular features, such as MMP-mediated collagen fragmentation, the primary sources (epidermis vs. dermis) and cell types (keratinocytes vs. fibroblasts) of elevated MMPs are different. Also, our investigations appear to reveal that collagen fragmentation in aged skin dermis results from constitutive 
Fig 4. Reduced fibroblast size upregulates c-Jun/c-Fos and activates AP-1, the major regulator of multiple matrix metalloproteinases (MMPs). Dermal fibroblasts from young skin ( $26 \pm 3$ years) were cultured in three-dimensional collagen lattices or monolayer under conditions of reduced cell size, as described in the Materials and methods section. (a) Reduction of fibroblast size upregulates c-Jun mRNA expression. (b) Reduction of fibroblast size upregulates c-Jun protein expression. (c) Reduction of fibroblast size upregulates c-Fos mRNA expression. (d) Reduction of fibroblast size upregulates c-Fos protein expression. mRNA levels were quantified by real-time reverse transcription polymerase chain reaction and were normalized by the housekeeping gene (36B4, internal control). Data are mean \pm SEM $(\mathrm{n}=3) . * \mathrm{P}<0.05$. Protein levels were determined by ProteinSimple (Santa Clare, CA, U.S.A.) capillary electrophoresis immunoassay (see Materials and methods section for details) and normalized by $\beta$-actin (loading control). Insert shows representative digital images. Data are mean \pm SEM $(\mathrm{n}=3) . * \mathrm{P}<0 \cdot 05$. (e) Dermal fibroblasts were transfected with AP-1 reporter construct (pAP-1-TA-Luc) or empty vector (CTRL). Cell lysates were prepared $48 \mathrm{~h}$ after transfection. Reporter activity was determined by luciferase assay. Data are mean $\pm \operatorname{SEM}(\mathrm{n}=3) . * \mathrm{p}<0.05$.
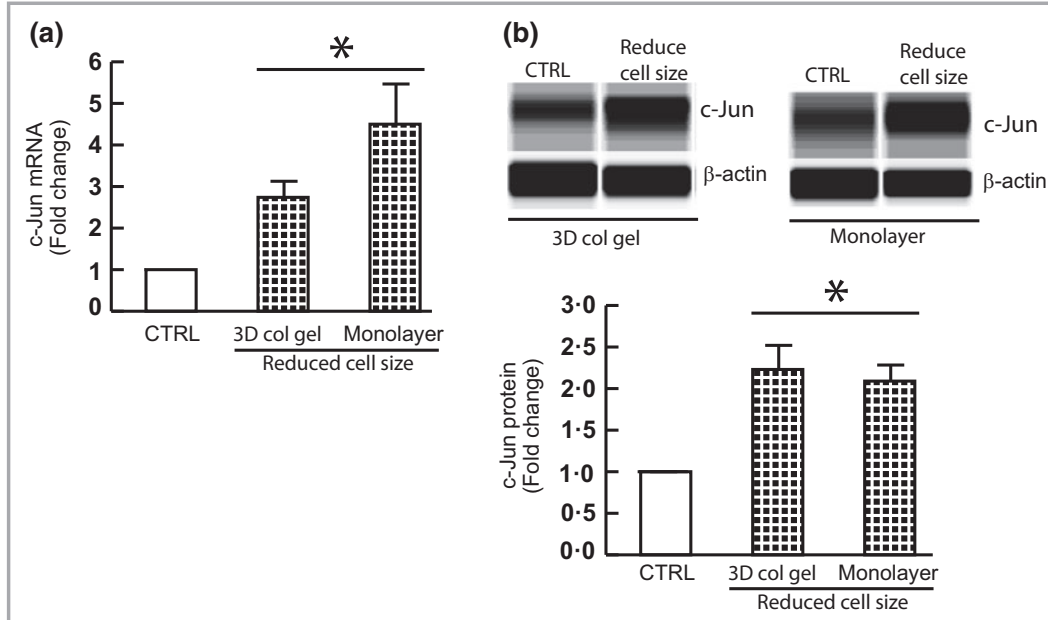

(c)

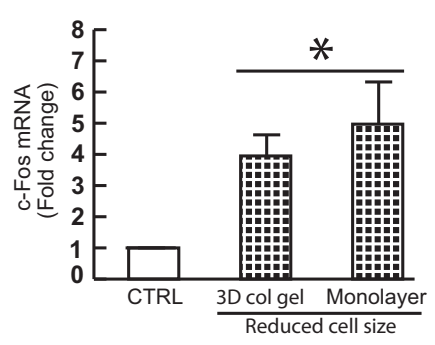

(d)

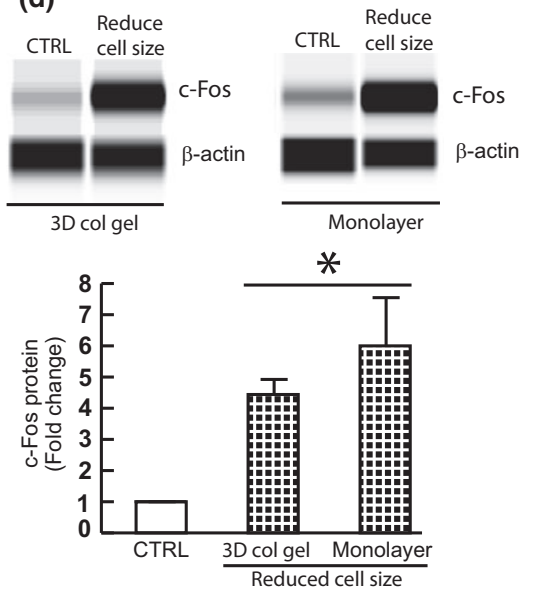

(d)

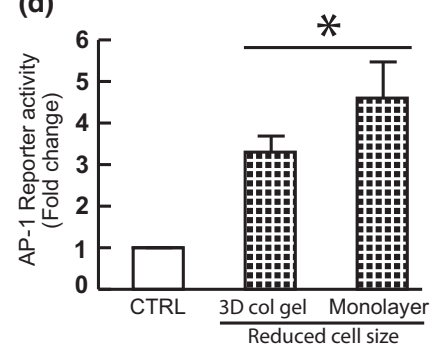

elevation of a wide variety of dermal MMPs, rather than a transient elevation of limited epidermal MMPs, as observed in acute UV irradiation in human skin.

Dermal fibroblasts are responsible for the production of these MMPs, and dermal fibroblasts are the major MMP-producing cells and control collagen homeostasis in the dermis. We demonstrate here that reduced fibroblast size upregulates multiple MMPs as observed in aged human skin dermis in vivo. Reduced fibroblast size is a prominent feature of dermal fibroblasts in aged human skin. ${ }^{3,16,17}$ We found that isolated primary dermal fibroblasts from aged ( $>80$ years) or young (25-30 years) individuals are indistinguishable with respect to morphology (Fig. 2d) and expression of all known mammalian MMPs (Fig. 2a). In contrast, human dermal fibroblasts, obtained from individuals of any age (21-86 years), cultured in the conditions of reduced cell size, result in the elevation of multiple MMPs (Table 1). These data suggest that the elevation of multiple MMPs in aged human skin arises, at least in part, from the reduced size of dermal fibroblasts. We are unable to confirm the fibroblast-size-dependent increase of MMP-23 and MMP-24, which are elevated in aged human skin dermis. As both MMP-23 and MMP-24 are cell membrane-associated MMPs (type II transmembrane MMPs), we found that the basal mRNA expression levels of these two MMPs are extremely low in dermal fibroblasts, suggesting that dermal fibroblasts are not a major source of elevated MMP-23 and MMP-24 in aged human skin dermis.

Further investigation indicates that reduced fibroblast size is closely associated with elevated c-Jun/c-Fos and increased transcription factor AP-1 activity, the major driving force for 


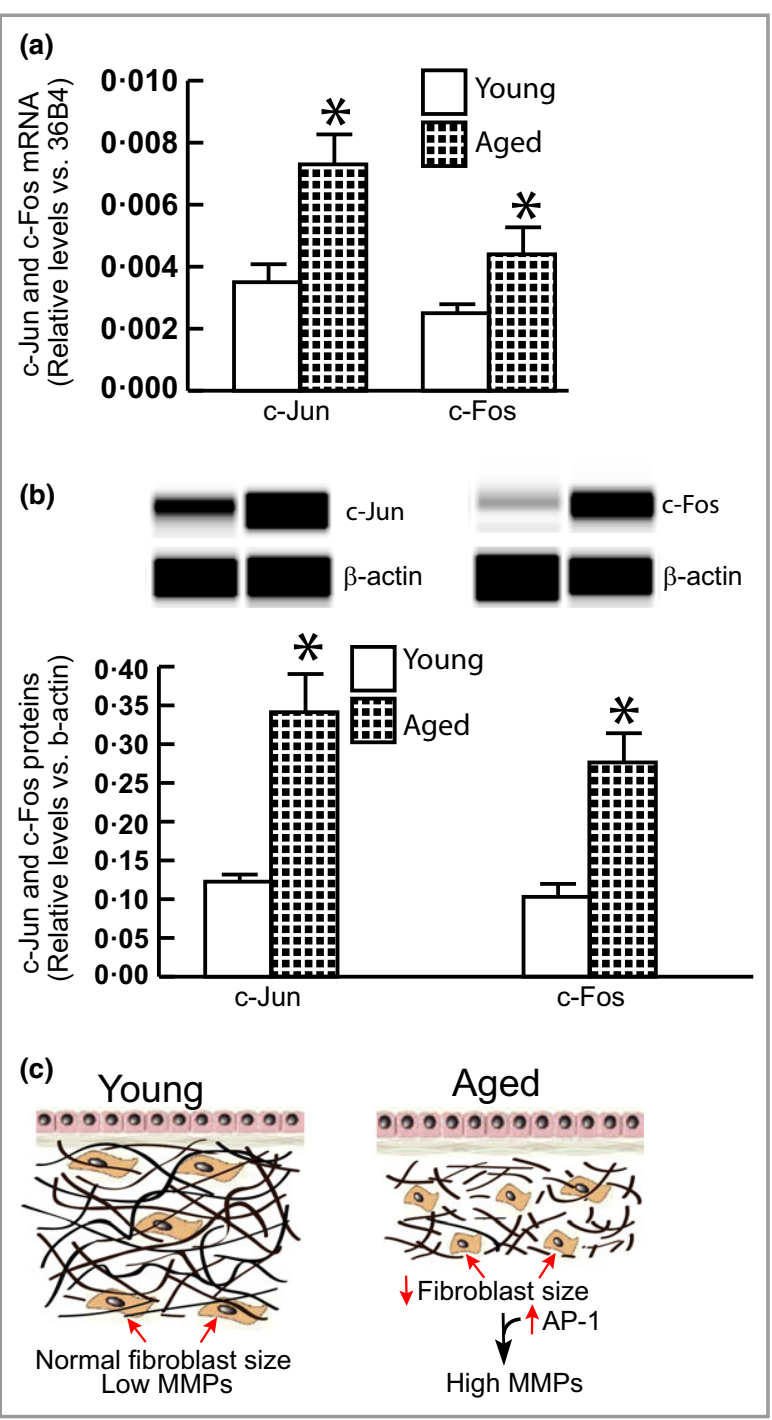

Fig 5. Elevated expression of c-Jun and c-Fos expression in aged human skin dermis. Adult human skin punch biopsies were obtained from young ( $26 \pm 3$ years) and aged ( $83 \pm 4$ years) sun-protected buttock skin. Dermis was prepared by cutting off epidermis at a depth of $1 \mathrm{~mm}$ by cryostat. (a) Total RNA was prepared from dermis and mRNA levels were quantified by real-time reverse transcription polymerase chain reaction and were normalized by the housekeeping gene (36B4, internal control), mean $\pm \operatorname{SEM}(n=6) .{ }^{*} \mathrm{P}<0.05$. (b) Dermal protein levels were determined by ProteinSimple (Santa Clare, CA, U.S.A.) capillary electrophoresis immunoassay (see Materials and methods section for details) and normalized by $\beta$-actin (loading control). Insert shows representative digital images. Data are mean $\pm \operatorname{SEM}(n=6) . * P<0.05$. (c) Reduced fibroblast size elevates age-related matrix metalloproteinases (MMPs) (see Discussion section for details).

multiple MMPs. ${ }^{21,22,27}$ Transcription factor AP-1, typically composed of c-Jun and C-Fos, is one of the first mammalian transcription factors to be identified. ${ }^{31,32}$ In addition to reports by other authors, we have previously reported that stress-activated MAP kinase pathways and c-Jun mRNA and protein are increased in aged human skin, compared with young human skin in vivo. ${ }^{17,33,34}$ In this study, we confirmed that c-Jun and c-Fos expression are significantly elevated in aged human skin dermis in vivo, which is the major source of elevated multiple MMPs. These data suggest that reduced fibroblast size induces c-Jun and c-Fos, which in turn elevates multiple MMPs in aged human skin dermis.

In skin dermis, fibroblast spreading and size, which are mediated by cytoskeletal and intracellular structural machinery, largely depend on cellular interactions with the surrounding ECM microenvironment. In young human skin dermis, the binding of fibroblasts to intact collagen fibrils allows for the generation of traction forces that are necessary for spreading, mechanical stability and normal function. However, in aged human skin dermis, collagen fibril binding sites are lost and mechanical resistance to traction forces is reduced owing to fragmentation. In this state, the ECM microenvironment is unable to provide sufficient mechanical stability to maintain normal cell spreading/mechanical force. ${ }^{3,16,17}$ Therefore, agerelated fragmentation of the collagen fibril microenvironment deleteriously alters fibroblast size and function.

Also, cell shape and size impacts multiple cellular processes including signal transduction, gene expression and metabolism. ${ }^{14,35-37}$ Currently, the mechanisms by which reduced cell size induces c-Jun/AP-1 are not well understood. AP-1 activity is regulated by a wide range of stimuli, including reactive oxygen species (ROS) ${ }^{32}$ We previously reported that fibroblasts with reduced spreading/size as a result of the fragmentation of surrounding collagen fibrils display increased levels of ROS. ${ }^{17}$ ROS is considered to be a major driving force of the ageing process. ${ }^{38,39}$ Indeed, we observed that protein oxidation, which is a marker of oxidative stress, is increased in aged human dermis in vivo. ${ }^{17}$ These data suggest that elevated ROS may mediate induction of c-Jun/AP-1 activity in response to reduced fibroblast spreading and size. Clearly, further studies are needed to understand the connections between fibroblast size, oxidative stress, AP-1 activity and MMPs.

We have previously reported that the reduction of fibroblast size by the disruption of the cytoskeleton reduced cellular mechanical force, which substantially induced MMP-1 expression $^{40}$ and consequent collagen fibril fragmentation. ${ }^{17}$ The reduced mechanical force induced transcription factor c-Jun to bind to a canonical AP-1 binding site in the MMP-1 proximal promoter. Blocking c-Jun function with dominant negative mutant c-Jun significantly reduced the induction of MMP-1 expression in response to reduced cell size and mechanical force. This current study added new information indicating that reduced fibroblast size not only induces MMP1, but also induces multiple MMPs (Fig. 1a). Furthermore, we demonstrated that the restoration of fibroblast size reversed elevated multiple MMPs to a basal level (Fig. 3f). We also previously reported that reduced fibroblast size inhibits type I procollagen synthesis, the major structural protein in skin, via impairment of transforming growth factor (TGF)$\beta /$ Smad signalling. ${ }^{41}$ Further investigation has revealed that reduced fibroblast size specifically downregulates TGF- $\beta$ type II receptor, and this downregulation largely mediates the 
reduction of type I procollagen and other ECM production. Taken together, these data suggest that reduced fibroblast size could be a marker of cellular ageing and of ageing human skin dermis.

Impaired wound healing is common in the elderly and presents a significant clinical and economic problem. It has been reported that fibroblast dysfunction is a key factor in the nonhealing of chronic wounds in the elderly. ${ }^{42,43}$ Dermal fibroblasts are largely responsible for collagen turnover and remodelling in the healing of wounds. Although MMPs play essential and beneficial roles in normal wound healing, MMPs must be present at the right amount and in the right time frame (duration) during the wound-healing process. There is substantial evidence that MMPs in general are highly elevated in delayed wound healing ${ }^{44-47}$ and that treatments that lower MMP activity accelerate the healing of wounds. ${ }^{48-50}$ It is conceivable that constitutive elevations of multiple MMPs, as identified in the current paper, result in a hostile tissue microenvironment that promotes age-related skin diseases, such as delayed wound healing in the elderly. Clearly, additional studies are warranted to uncover the precise molecular mechanism(s) by which constitutive elevations of multiple MMPs may contribute to impaired wound healing in the elderly.

In summary, aged dermis constitutively expresses elevated levels of several MMPs, which likely lead to chronic, progressive degradation of the dermal collagen in aged human skin. We propose a novel mechanism by which age-related reduction of fibroblast size activates AP-1, which in turn elevates multiple MMPs as observed in aged human skin (Fig. 5c). This mechanism provides a foundation for understanding the cellular and molecular basis of age-related collagen fragmentation, the characteristic feature of aged human skin.

\section{Acknowledgments}

We thank Ken Calderone, Yuan Shao and Patrick Robichaud for technical assistance and Diane Fiolek for graphics and administrative assistance. This work was supported by the National Institute of Health (AG19364 to G.F. and T.Q.).

\section{Author contributions}

Z.Q. and R.M.B. performed the experiments. T.Q. designed the study, analysed the data and wrote the manuscript.

\section{References}

1 Di Lullo GA, Sweeney SM, Korkko J et al. Mapping the ligandbinding sites and disease-associated mutations on the most abundant protein in the human, type I collagen. J Biol Chem 2002; 277:4223-31.

2 Uitto J. Connective tissue biochemistry of the aging dermis. Agerelated alterations in collagen and elastin. Dermatol Clin 1986; 4:433-46.

3 Fisher GJ, Varani J, Voorhees JJ. Looking older: fibroblast collapse and therapeutic implications. Arch Dermatol 2008; 144:666-72.
4 Quan T, Fisher GJ. Role of age-associated alterations of the dermal extracellular matrix microenvironment in human skin aging: a mini-review. Gerontology 2015; 61:427-34.

5 Ma W, Wlaschek M, Tantcheva-Poór I et al. Chronological ageing and photoageing of the fibroblasts and the dermal connective tissue. Clin Exp Dermatol 2001; 26:592-9.

6 Ashcroft GS, Horan MA, Ferguson MW. The effects of ageing on cutaneous wound healing in mammals. J Anat 1995; 187:1-26.

7 Gilchrest BA, Stoff JS, Soter NA. Chronologic aging alters the response to ultraviolet-induced inflammation in human skin. J Invest Dermatol 1982; 79:11-5.

8 Eaglstein WH. Wound healing and aging. Clin Geriatr Med 1989; 5:183-8.

9 Ashcroft GS, Mills SJ, Ashworth JJ. Ageing and wound healing. Biogerontology 2002; 3:337-45.

10 Rogers HW, Weinstock MA, Feldman SR, Coldiron BM. Incidence estimate of nonmelanoma skin cancer (keratinocyte carcinomas) in the US population, 2012. JAMA Dermatol 2015; 151:1081-6.

11 Pickup MW, Mouw JK, Weaver VM. The extracellular matrix modulates the hallmarks of cancer. EMBO Rep 2014; 15:1243-53.

12 Quan T. Skin connective tissue aging and dermal fibroblasts. In: Dermal Fibroblasts: Histological Perspectives, Characterization and Role in Disease (Bai X ed.). Hauppauge, NY: Nova Biomedical, 2013; 31-55.

13 Hynes RO. The extracellular matrix: not just pretty fibrils. Science 2009; 326:1216-9.

14 Ingber DE. Cellular mechanotransduction: putting all the pieces together again. FASEB J 2006; 20:811-27.

15 Geiger B, Spatz JP, Bershadsky AD. Environmental sensing through focal adhesions. Nat Rev Mol Cell Biol 2009; 10:21-33.

16 Varani J, Schuger L, Dame MK et al. Reduced fibroblast interaction with intact collagen as a mechanism for depressed collagen synthesis in photodamaged skin. J Invest Dermatol 2004; 122:1471-9.

17 Fisher GJ, Quan T, Purohit T et al. Collagen fragmentation promotes oxidative stress and elevates matrix metalloproteinase- 1 in fibroblasts in aged human skin. Am J Pathol 2009; 174:101-14.

18 Bonnans C, Chou J, Werb Z. Remodelling the extracellular matrix in development and disease. Nat Rev Mol Cell Biol 2014; 15:786-801.

19 Hegedus L, Cho H, Xie X, Eliceiri GL. Additional MDA-MB-231 breast cancer cell matrix metalloproteinases promote invasiveness. J Cell Physiol 2008; 216:480-5.

20 Qin Z, Fisher GJ, Quan T. Cysteine-rich protein 61 (CCN1) domain-specific stimulation of matrix metalloproteinase-1 expression through $\alpha \mathrm{V} \beta 3$ integrin in human skin fibroblasts. J Biol Chem 2013; 288:12386-94.

21 Chakraborti S, Mandal M, Das S et al. Regulation of matrix metalloproteinases: an overview. Mol Cell Biochem 2003; 253:269-85.

22 Gutman A, Wasylyk B. The collagenase gene promoter contains a TPA and oncogene-responsive unit encompassing the PEA3 and AP-1 binding sites. EMBO J 1990; 9:2241-6.

23 Birkedal-Hansen H, Moore WG, Bodden MK et al. Matrix metalloproteinases: a review. Crit Rev Oral Biol Med 1993; 4:197-250.

24 Fisher GJ, Wang ZQ, Datta SC et al. Pathophysiology of premature skin aging induced by ultraviolet light. N Engl J Med 1997; 337:1419-28.

25 Quan T, Qin Z, Voorhees JJ, Fisher GJ. Cysteine-rich protein 61 (CCN1) mediates replicative senescence-associated aberrant collagen homeostasis in human skin fibroblasts. J Cell Biochem 2012; 113:3011-8.

26 Gieni RS, Hendzel MJ. Mechanotransduction from the ECM to the genome: are the pieces now in place? J Cell Biochem 2008; 104: $1964-87$.

27 Benbow U, Brinckerhoff CE. The AP-1 site and MMP gene regulation: what is all the fuss about? Matrix Biol 1997; 15:519-26. 
28 Brenneisen P, Sies H, Scharffetter-Kochanek K. Ultraviolet-B irradiation and matrix metalloproteinases: from induction via signaling to initial events. Ann N Y Acad Sci 2002; 973:31-43.

29 Fisher GJ, Datta SC, Talwar HS et al. Molecular basis of suninduced premature skin ageing and retinoid antagonism. Nature 1996; 379:335-9.

30 Quan T, Qin Z, Xia W et al. Matrix-degrading metalloproteinases in photoaging. J Investig Dermatol Symp Proc 2009; 14:20-4.

31 Angel P, Karin M. The role of Jun, Fos and the AP-1 complex in cell-proliferation and transformation. Biochim Biophys Acta 1991; 1072:129-57.

32 Shaulian E, Karin M. AP-1 as a regulator of cell life and death. Nat Cell Biol 2002; 4:e131-6.

33 Chung JH, Kang S, Varani J et al. Decreased extracellular-signalregulated kinase and increased stress-activated MAP kinase activities in aged human skin in vivo. J Invest Dermatol 2000; 115: 177-82.

34 Shin $\mathrm{MH}$, Rhie GE, Kim YK et al. H2O2 accumulation by catalase reduction changes MAP kinase signaling in aged human skin in vivo. J Invest Dermatol 2005; 125:221-9.

35 Alenghat FJ, Nauli SM, Kolb R et al. Global cytoskeletal control of mechanotransduction in kidney epithelial cells. Exp Cell Res 2004; 301:23-30

36 Silver FH, Siperko LM, Seehra GP. Mechanobiology of force transduction in dermal tissue. Skin Res Technol 2003; 9:3-23.

37 Wang N, Butler JP, Ingber DE. Mechanotransduction across the cell surface and through the cytoskeleton. Science 1993; 260:1124-7.

38 Stadtman ER. Protein oxidation and aging. Science 1992; 257: $1220-4$.

39 Golden TR, Hinerfeld DA, Melov S. Oxidative stress and aging: beyond correlation. Aging Cell 2002; 1:117-23.

40 Qin Z, Voorhees JJ, Fisher GJ, Quan T. Age-associated reduction of cellular spreading/mechanical force up-regulates matrix metalloproteinase-1 expression and collagen fibril fragmentation via
c-Jun/AP-1 in human dermal fibroblasts. Aging Cell 2014; 13:1028-37.

41 Fisher GJ, Shao Y, He T et al. Reduction of fibroblast size/mechanical force down-regulates TGF-beta type II receptor: implications for human skin aging. Aging Cell 2016; 15:67-76.

42 Mendez MV, Stanley A, Park HY et al. Fibroblasts cultured from venous ulcers display cellular characteristics of senescence. J Vasc Surg 1998; 28:876-83.

43 Wall IB, Moseley R, Baird DM et al. Fibroblast dysfunction is a key factor in the non-healing of chronic venous leg ulcers. J Invest Dermatol 2008; 128:2526-40.

44 Wysocki AB, Staiano-Coico L, Grinnell F. Wound fluid from chronic leg ulcers contains elevated levels of metalloproteinases MMP-2 and MMP-9. J Invest Dermatol 1993; 101:64-8.

45 Weckroth M, Vaheri A, Lauharanta J et al. Matrix metalloproteinases, gelatinase and collagenase, in chronic leg ulcers. J Invest Dermatol 1996; 106:1119-24.

46 Lobmann R, Ambrosch A, Schultz G et al. Expression of matrixmetalloproteinases and their inhibitors in the wounds of diabetic and non-diabetic patients. Diabetologia 2002; 45:1011-6.

47 Yager DR, Zhang LY, Liang HX et al. Wound fluids from human pressure ulcers contain elevated matrix metalloproteinase levels and activity compared to surgical wound fluids. J Invest Dermatol 1996; 107:743-8.

48 Veves A, Sheehan P, Pham HT. A randomized, controlled trial of Promogran (a collagen/oxidized regenerated cellulose dressing) vs. standard treatment in the management of diabetic foot ulcers. Arch Surg 2002; 137:822-7.

49 Cullen B. The role of oxidized regenerated cellulose/collagen in chronic wound repair. Part 2. Ostomy Wound Manage 2002; 48:8-13.

50 Lobmann R, Zemlin C, Motzkau M et al. Expression of matrix metalloproteinases and growth factors in diabetic foot wounds treated with a protease absorbent dressing. J Diabetes Complications 2006; 20:329-35. 